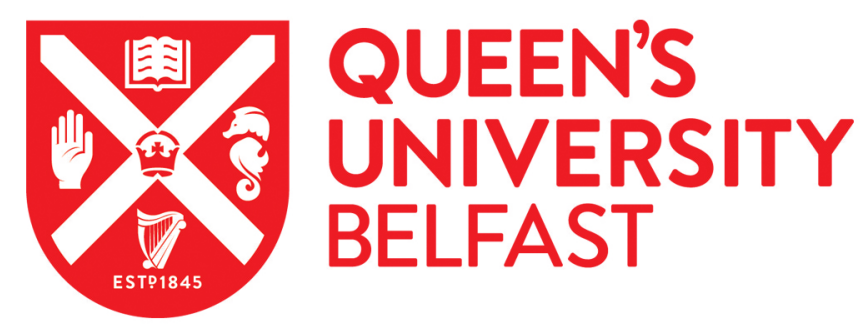

\title{
Infection with Prevotella nigrescens induces TLR2 signalling and low levels of p65 mediated inflammation in Cystic Fibrosis bronchial epithelial cells
}

Bertelsen, A., Elborn, J., \& Schock, B. (2019). Infection with Prevotella nigrescens induces TLR2 signalling and low levels of p65 mediated inflammation in Cystic Fibrosis bronchial epithelial cells. Journal of cystic fibrosis : official journal of the European Cystic Fibrosis Society. https://doi.org/10.1016/j.jcf.2019.09.005

\section{Published in:}

Journal of cystic fibrosis : official journal of the European Cystic Fibrosis Society

\section{Document Version:}

Peer reviewed version

Queen's University Belfast - Research Portal:

Link to publication record in Queen's University Belfast Research Portal

\section{Publisher rights}

Copyright 2019 Elsevier.

This manuscript is distributed under a Creative Commons Attribution-NonCommercial-NoDerivs License

(https://creativecommons.org/licenses/by-nc-nd/4.0/), which permits distribution and reproduction for non-commercial purposes, provided the author and source are cited.

\section{General rights}

Copyright for the publications made accessible via the Queen's University Belfast Research Portal is retained by the author(s) and / or other copyright owners and it is a condition of accessing these publications that users recognise and abide by the legal requirements associated with these rights.

Take down policy

The Research Portal is Queen's institutional repository that provides access to Queen's research output. Every effort has been made to ensure that content in the Research Portal does not infringe any person's rights, or applicable UK laws. If you discover content in the

Research Portal that you believe breaches copyright or violates any law, please contact openaccess@qub.ac.uk. 
1 Infection with Prevotella nigrescens induces TLR2 signalling and low levels of p65 mediated

2 inflammation in Cystic Fibrosis bronchial epithelial cells

3

$4 \quad$ A. Bertelsen*,\#, J.S. Elborn ${ }^{\#,++}$, B.C. Schock ${ }^{\#, S}$

5

6

* Department of Veterinary Medicine, The University of Cambridge, Madingley Road, Cambridge,

7 United Kingdom.

8 "Wellcome-Wolfson Institute for Experimental Medicine, Queens University Belfast, Belfast, United 9 Kingdom.

$10 \quad{ }^{++}$National Heart and Lung Institute, Imperial College London, London, United Kingdom.

11

12 Corresponding author contact details:

13 Queens University Belfast, Wellcome-Wolfson Institute for Experimental Medicine, Medical Biology

14 Building, 97 Lisburn Road, Belfast BT9 6BL, UK

15 Phone: +44 2890972258

16 Email: b.schock@qub.ac.uk

21 Keywords:

22 Cystic Fibrosis Airways inflammation, Anaerobic bacteria, Prevotella nigrescens, Host-pathogen-

23 Interaction, Signalling

26 Running title:

27 P.nigrescens signalling in CF airway 
29 Prevotella spp. are frequently identified in Cystic Fibrosis sputum. This study examined whether 30 infection with Prevotella nigrescens, a frequently identified member of this species, contributes to 31 inflammation in CF bronchial epithelial cells through activation of TLR- and NF-KB signalling pathways.

32 CFBE410- cells were infected with either P.nigrescens or Pseudomonas aeruginosa and incubated 33 under anaerobic conditions for 4 hours. P.nigrescens activated TLR2 signalling but not TLR4 signalling 34 while P.aeruginosa activated TLR4 signalling with a lesser effect on TLR2. P.aeruginosa induced significant $\mathrm{IKB} \alpha$ phosphorylation 10 minutes post infection with a return to control levels by 30 minutes post infection. A significant induction in nuclear p65 DNA binding was observed at 2 hours post infection. In contrast, infection with P.nigrescens induced phosphorylation of

38 І $\mathrm{KB} \alpha 120$ minutes post infection, with significant induction in nuclear p65 DNA binding at 4 hours post

39 infection only. Cytokine gene and protein responses were lower for P.nigrescens compared to 40 P.aeruginosa.

41 This study demonstrates the ability of a clinical P.nigrescens isolate to provoke a delayed NF-kB(p65) 42 driven response through induction in TLR2 signalling and activation of sustained levels of IKK $\alpha$. 
Therapeutic advances combined with multi-disciplinary care have dramatically increased life expectancy for people with Cystic Fibrosis (PWCF) and infants born today are predicted to survive for almost 50 years ${ }^{1}$. Such therapeutic approaches are helping drive targeted therapies to prevent disease progression ${ }^{2,3}$. However, although current potentiator/corrector drugs improve the expression and function of the Cystic Fibrosis conductance Transmembrane Regulator (CFTR), they fail to reduce sputum inflammatory markers ${ }^{4,5}$. Thus, the primary cause of mortality in PWCF is still progressive lung damage, caused by bacterial infection and subsequent inflammation in the lung ${ }^{3}$. Consequently, in order to prevent excessive infection and lung damage, there is still a need to enhance the understanding of pathogen-host interactions in CF airways. Bacterial recognition by Toll-Like Receptors (TLRs) results in activation of canonical nuclear factor kappa-light-chain-enhancer of activated B cells (NF-kB) signalling in mammalian cells, and subsequent release of inflammatory cytokines and chemokines ${ }^{6,7}$. Induction of these pathways requires the adaptor proteins myeloid differentiation primary response 88 (MYD88), Interleukin-1 Receptor (IL-1R) associated kinases (IRAK), inhibitor of kappa B (IKB $\alpha$ ) and translocation of the NF-?? subunits p65/p50 into the nucleus. Resolution of the cascade is complex and depends on newly synthesized IKBa to localise $p 65$ back to the cytoplasm, the zinc-finger protein A20 to eliminate TNF receptor associated factor 6 (TRAF6) activation, and the induction of IKB kinase $\alpha$ (IKK $\alpha$ ), which promotes p65 and c-Rel turnover and removal in TLR expressing cells ${ }^{8-10}$. P.aeruginosa is a major contributor to inflammation in the CF lung, together with other well-studied organisms causative of inflammatory responses in airway cells in PWCF such as Staphylococcus aureus, Haemophilus influenzae and non-fermenting Gram-negative bacteria such as Burkholderia cepecia complex ${ }^{3}$.

P.aeruginosa infections are dynamic and complex. P. aeruginosa can activate TLR4 (through LPS) and TLR5 (through flagellin) and driving a TLR/NF-KB-dependent inflammatory cascade in bronchial epithelial cells, resulting in secretion of elevated levels of IL-8, further contributing to the variety of inflammatory responses in CF lungs ${ }^{11,12}$. In mouse lungs, flagellin production gave $P$.aeruginosa a fitness disadvantage, while flagella-negative strains had enhanced fitness ${ }^{13}$. From the host's point, a

83 TLR-based response to a pathogen is the desired event to promote the elimination of the pathogen ${ }^{14}$, 84 but in chronic infection of CF airways in vivo, in addition to the loss of TLR5-activating flagellin, 85 P.aeruginosa modifies its LPS (lipid A) causing less TLR4 activation ${ }^{15}, 16$, and it has been suggested that 
86 other well-studied P.aeruginosa virulence factors (e.g. exopolysaccharide, avoiding adaptive

87 immunity) may also contribute to the progression of CF lung disease ${ }^{17}$.

88 Molecular techniques based on next generation pyrosequencing have identified a diverse microbiome

89 in the lungs of healthy volunteers and PWCF. Such microbiomes include a number of anaerobic Gram-

90 negative Prevotella species; however few studies have explored the biological role of this species in

91 contributing to infection and inflammatory responses in the lungs of PWCF ${ }^{18-21}$. P. nigrescens is an

92 exemplar of this species and has been identified by culture and sequencing in sputum obtained from

93 PWCF sputum ${ }^{22}$.

94 Previous studies utilising heat killed bacteria, bacterial lysates and supernatants have shown that $P$.

95 nigrescens possesses the ability to degrade host anti-proteases and suppress IL-4 responses in

96 mammalian and murine experimental systems, provoking a range of inflammatory responses ${ }^{23-25}$.

97 However, currently no studies have examined the role of live P.nigrescens on inflammatory responses

98 in airway cells in PWCF. We hypothesized that the presence of P.nigrescens in the lung of PWCF may

99 contribute to the elevated levels of inflammatory responses observed in CF airway epithelial cells and

100 aimed to investigate the role of TLR and nuclear NF-KB signalling pathways involved in this response.

101

102 
105 We have used clinical isolates obtained from a patient attending the adult CF clinic at Belfast City

106 Hospital throughout the study. The two isolates studied here were derived from the same patient:

107 P.nigrescens isolate B021V1S1 (B = Belfast, 021= patient, V1S1 = first visit stable sample, herein

108 abbreviated B021S) was grown under anaerobic conditions for 72 hours using a Don Whitley anaerobic

109 cabinet as described previously ${ }^{9}$. The P.nigrescens strain utilised was P.nigrescens B021S identified

110 through the use of $16 \mathrm{~S}$ rRNA sequencing ${ }^{22}$. Further information given in the supplement.

111 P.aeruginosa isolate $\mathrm{B} 021$ was grown under aerobic conditions $\left(37^{\circ} \mathrm{C}, 5 \% \mathrm{CO}_{2}\right)$ for 24 hours before

112 infection of cells. This culture was used to inoculate a liquid culture of Lysogeny Broth, which was

113 incubated for 2 hours under aerobic conditions at $200 \mathrm{rpm}$ before being used to infect cells.

\section{Cell culture}

115 A Cystic Fibrosis bronchial epithelial cell line homozygous for Phe508del (CFBE410-, a kind gift from D

116 Gruenert, USF) was maintained in the absence of antibiotic supplementation under standard cell

117 culture conditions $\left(37^{\circ} \mathrm{C}, 5 \% \mathrm{CO}_{2}, 95 \%\right.$ mixed gas). Cells were passaged as described previously (7).

118 HEK-293-TLR2, HEK-293-TLR4, HEK-293-TLR5 and HEK-293-TLR null cells were maintained as per

119 manufacturer's instructions (InvivoGen). Once infected with the clinical isolates of P.aeruginosa or

120 P.nigrescens, cells were incubated anaerobically for the duration of the experiments (up to $4 \mathrm{~h}$ ).

\section{Inflammatory studies}

122 CFBE410- cells were seeded into 48, 24 and 6 well tissue culture plates (Nunc). At $80 \%$ confluency 123 media was removed and the cells were washed with phosphate buffered saline (PBS, Gibco) and pre-

124 warmed fresh media (MEM) was added to each cell population. Cells were infected with an MOI of 100

125 of either P.nigrescens or P.aeruginosa as defined by plating serial dilutions of each inoculum. Cells

126 were then incubated under anaerobic environments for the duration of the experiments. The MOI

127 used was based on preliminary studies obtaining maximal responses (IL-8, p65) while ensuring cell 128 viability (minimal MTT and LDH responses, no changes in cell morphology) to infections with either

129 P.nigrescens and P.aeruginosa. Detailed information can be found in supplementary data S1.

130 TLR Reporter assays

131 HEK-293-TLR2, HEK-293-TLR4, HEK-293-TLR5 and HEK-293-TLR null cells were transiently transfected 
132 with an NF-KB luciferase containing reporter construct (InvivoGen) and luciferase release from infected

133 and uninfected control cells was assessed 4 hours post infection as described previously ${ }^{26}$.

134 Cytoplasmic and nuclear fraction extraction for DNA binding ELISA

135 CFBE41o- cells were infected as described, and nuclear and cytoplasmic cell fractions were extracted

136 from infected and uninfected control cells for assessment of IKK $\alpha, I K B \alpha, p 65$ and p50 by sandwich

137 ELISA (TransAM ${ }^{\mathrm{TM}}$ Activ Motif IKB $\alpha$ function ELISA, Cell Signalling Technology PathScan ${ }^{\circledR}$ Phospho-IKK $\alpha$

138 (Ser 176/180), TransAM $^{\text {тм }}$ Activ Motif NF-KB family function ELISA.

139 Gene expression analysis

140 Innate immune response profilers were purchased from SA Biosciences. Total RNA was extracted from

141 infected and uninfected cells and genomic DNA was eliminated from each sample as per

142 manufacturer's instructions (SA Biosciences).

143 RT-PCR for TLR and Cytokine gene expression

144 Total RNA was extracted from infected cell and uninfected control cell populations using a combination

145 of Trizol reagent and column extraction (Thermo Fisher Scientific). qPCR analysis was carried out on an

146 MX300-P (Agilent technologies) using SYBR green primers (QuantiTect, Qiagen) and the SYBR Green

147 PCR master mix kit (Thermo Fisher Scientific).

149 siRNA knockdown

150 Reverse transfection was used in all siRNA assays. siRNA (Thermo Fisher Scientific) was diluted to a 151 working concentration of $20 \mathrm{pmol} / \mathrm{mL}$ and reverse transfection was carried out as per manufacturer's 152 instructions (Thermo Fisher Scientific). Target gene and protein expression were assessed at 24-48 153 hours post transfection by qPCR to confirm successful knockdown. Average knockdown was found to 154 be $>70 \%$. Further details can be found in the supplementary data S2.

156 Statistical analysis

157 All experiments were performed in duplicates and data of three independent replicates are expressed

158 as mean +/- standard deviation. Parametric tests including t-test to assess the null hypothesis, or 1-

159 Way ANOVA with Bonferroni post-test correction were used to assess any significant differences in the 160 mean values in the following experiments. Two-way ANOVA was used to assess the variation between 161 matched samples over time with Bonferroni correction. 


\section{NF-KB signalling in CFBE410- cells infected with P.nigrescens or P.aeruginosa}

164 NF-KB activation and cytokine gene expression were determined 2 and 4 hours after infection. In response to P.aeruginosa CFBE410- cells exhibited a significant induction in phosphorylated IKB $\alpha$ protein at 10 minutes (67\% increase) and a significant increase in nuclear p65 DNA binding at both time points analysed ( 2 and 4 h, 2.8- and 2-fold increase, respectively), (Figure 1A-1B). In keeping with the significant p65-DNA binding in response to P.aeruginosa, a 5-fold increase in IL-6 gene expression and an 11-fold increase in IL-8 gene expression were observed 4 hours post infection (Figures 1C-1D). When infected with P.nigrescens CFBE410- cells displayed 70\% increase in phosphorylated IKB $\alpha$ protein 2 hours post infection leading to a 2 -fold increase in nuclear p 65 observed at 4 hours post infection (Figures 1A, 1B). Significantly lower levels of IL-6 and IL-8 gene expression were observed in P.nigrescens infected cells compared to P.aeruginosa infected cells at 4 hours, however the 2 hour levels of IL-6 mRNA observed from cells infected with P.nigrescens were significantly higher when compared to baseline controls.

A

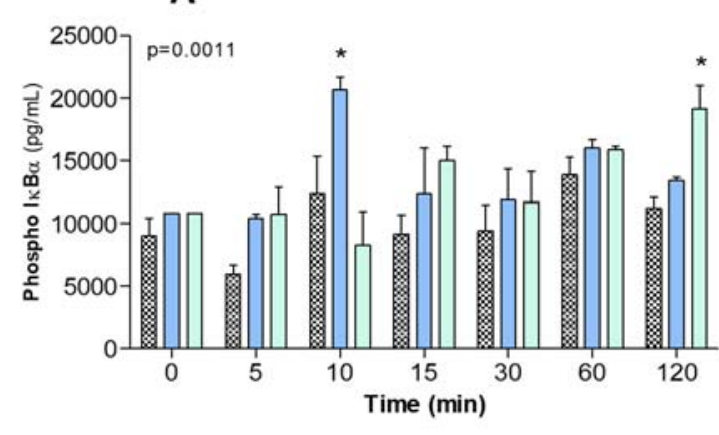

B

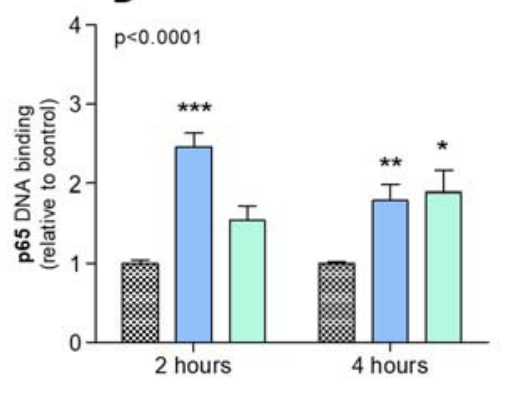

$\varpi$ Control $\square$ P.aeruginosa

$\square$ P.nigrescens
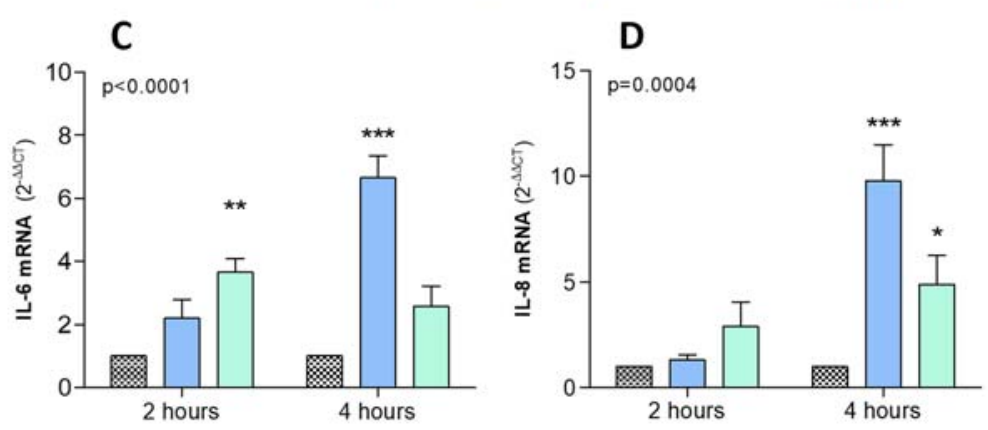

176

177

178

179

180

Figure 1: Inflammatory responses from CFBE410- cells infected with P.nigrescens or P.aeruginosa (MOI 100) and uninfected controls. 1A) Time course of IKB $\alpha$ phosphorylation; 1B) p65 DNA binding at 2 and 4 hours; 1C) IL-6 gene expression at 2- and 4-hour post infection and 1D) IL-8 gene expression at 2- and 4-hours post infection. All $n=3,{ }^{*} p<0.05,{ }^{* *} p<0.01,{ }^{* * *} p<0.001,2$-Way ANOVA with Bonferroni posttest, overall ANOVA given within the graph. 
182 HEK 293 reporter cell lines were infected with P.aeruginosa or P.nigrescens in order to determine if

183 infection with either isolate would activate TLR signalling.

184 A 20-fold induction in TLR2 signalling was observed at 4 hours post infection with $P$.aeruginosa while a

185 30- and 40-fold induction was observed at 2 and 4 hours post infection with P.nigrescens in HEK-293-

186 TLR2 cells. A 16-fold up regulation in TLR4 signalling was observed in HEK-293-TLR4 cells at 1 hour post

187 infection with P.aeruginosa and a 20-fold induction was observed at both 2 and 4 hours post infection

188 with P.aeruginosa in HEK-293-TLR4 cells (Figures 2A, 2B). An 11-fold induction in TLR5 signalling was

189 observed in HEK-293-TLR5 cells in response to infection with P.aeruginosa at 2 hours post infection and 190 a 19-fold induction was observed at 4 hours post infection (Figure 2C).

191

192

193

194

195

196

197

198

199

200

201

202

203

204

205

206

207
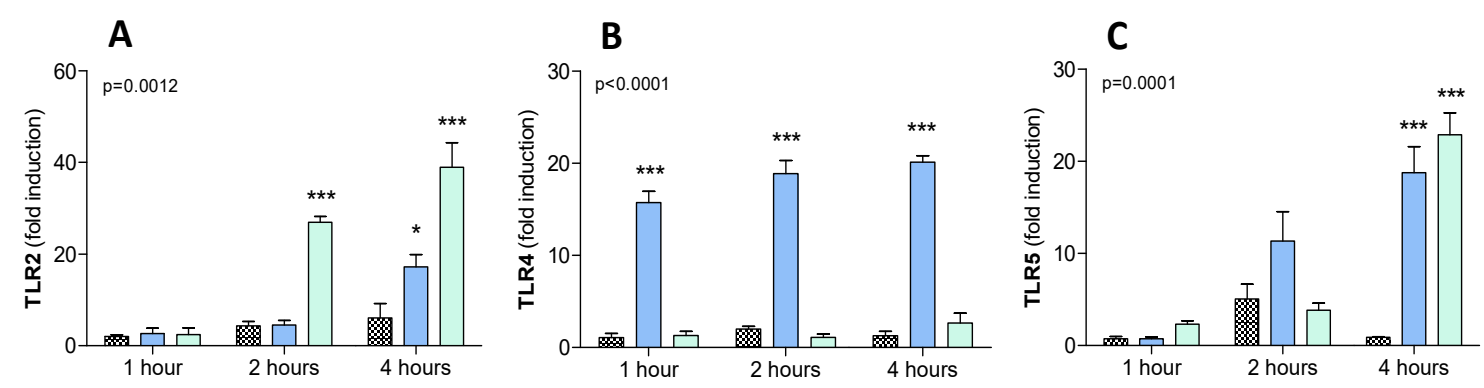

Fontrol

P.aeruginosa

P.nigrescens

Figure 2: TLR signalling using HEK-TLR reporter cell lines: 2A) HEK-293-TLR2; 2B) HEK-293-TLR4 and 2C) HEK-293-TLR5 signalling in response to infection with either P.nigrescens or P.aeruginosa (MOI 100). Infected cell populations were compared to non-infected cell populations. All $n=3,{ }^{*} p<0.05,{ }^{* *} p<0.01$, ${ }^{* * *} p<0.001,2$-Way ANOVA with Bonferroni post-test, overall ANOVA given within the graph.

TLR signalling activates p65 NF-KB signalling in CFBE410- cells infected with P.nigrescens. siRNA mediated knockdown of TLR2 and TLR5 in CFBE41o- cells confirmed that P.nigrescens infection activated TLR2 signalling in CFBE41o- cells and not TLR5 signalling, as measured by a $39 \%$ reduction in p65 DNA binding observed at 4 hours post infection in the TLR2 knockdown experiments (Figure 3B). IL-6 gene expression was reduced in TLR2 knockdown cells infected with P.nigrescens when compared to wild-type cells infected with P.nigrescens however this reduction was not statistically significant (Figure 3D). No significant decreases in IL-6 gene expression or the DNA binding abilities of nuclear p65 were observed in cells transfected with an siRNA targeted against TLR5 at any time point (Figures 3A, $3 C)$. 
A

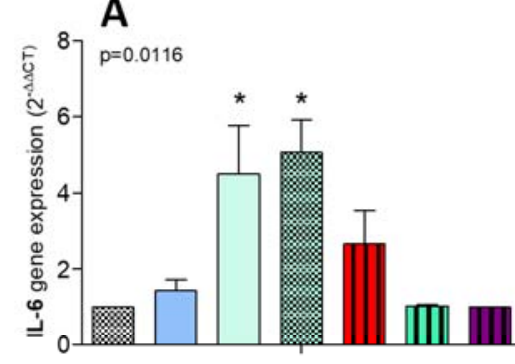

B

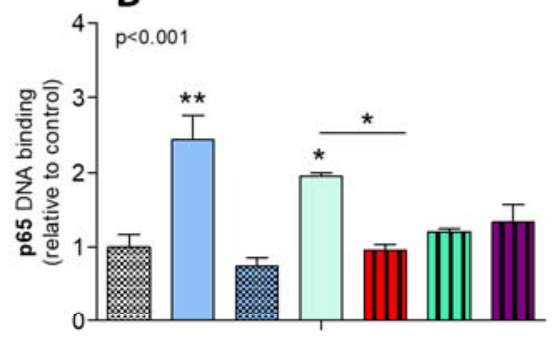

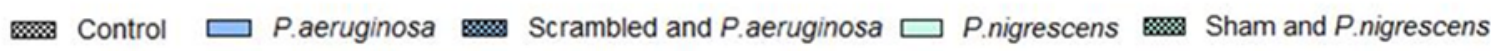
[u P.nigrescens and TLR2 siRNA

WI Scrambled siRNA
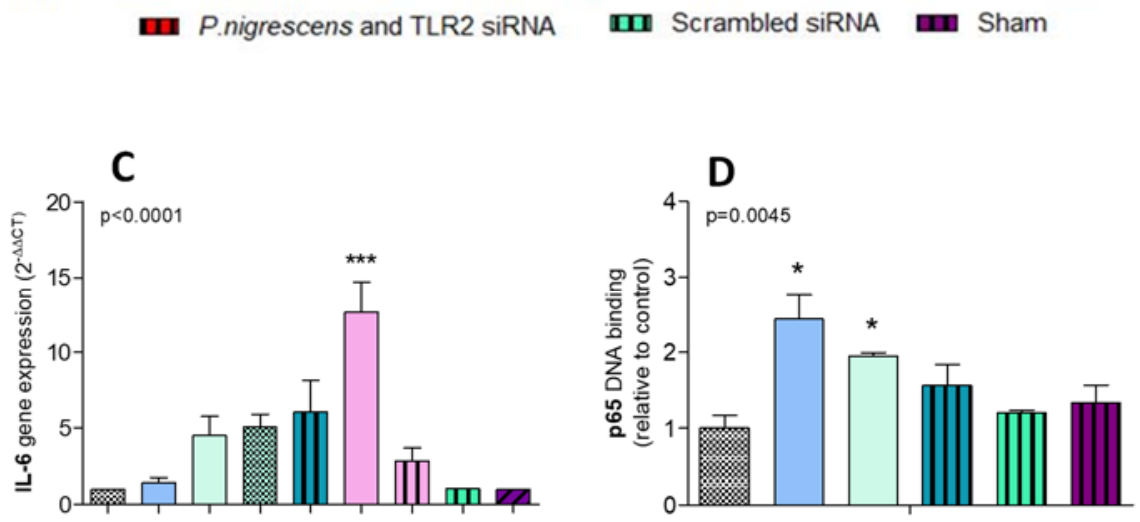

Figure 3: Effect of TLR2 and TLR5 knockdown on NF-kB induced inflammation in CFBE41o- cells. CFBE410- cells were infected with P.nigrescens or P.aeruginosa (MOI 100, 2 or 4 hours) and compared to non-infected and to wild-type P.nigrescens infected CFBE410- cells. Scrambled and sham transfections were compared to non-stimulated controls. 3A) IL-6 gene expression in response to TLR2 knockdown (MOI 100, 2 hours). 3B) p65 phosphorylation with TLR2 siRNA knockdown (MOI 100, 4 hours). 3C) IL-6 gene expression in response to TLR5 knockdown (MOI 100, 2 hours). 3D) p65 DNA binding in CFBE41o- cells with TLR5 siRNA knockdown (MOI 100, 4 hours). All $n=3,{ }^{*} p<0.05, * * p<0.01$, ${ }^{* * *} p<0.001$, 1-Way ANOVA with Bonferroni post-test, overall ANOVA given within the graph.

The contribution of IKKa to inflammation induced by infection by P.nigrescens in CF bronchial epithelial cells.

CFBE410- cells displayed sustained 3-fold induction in IKK $\alpha$ phosphorylation in response to infection with $P$.nigrescens as measured at $\mathrm{t}=0-60$ minutes (Figure 4A). P.aeruginosa infection, in contrast, only resulted in a 2-fold increase in IKK $\alpha$ phosphorylation 60 minutes post infection (Figure 4A). siRNA mediated knockdown of IKK $\alpha$ resulted in the restoration of nuclear p65 DNA binding at 2 hours in cells infected with P.nigrescens. A 2.7-fold induction in nuclear p65 DNA binding was observed in CF cells transfected with siRNA targeted against IKK $\alpha$ and infected with P.nigrescens suggesting a role for IKK $\alpha$ in repressing inflammation in CF bronchial epithelial cells in response to infection with P.nigrescens (Figure 4 B). 
A

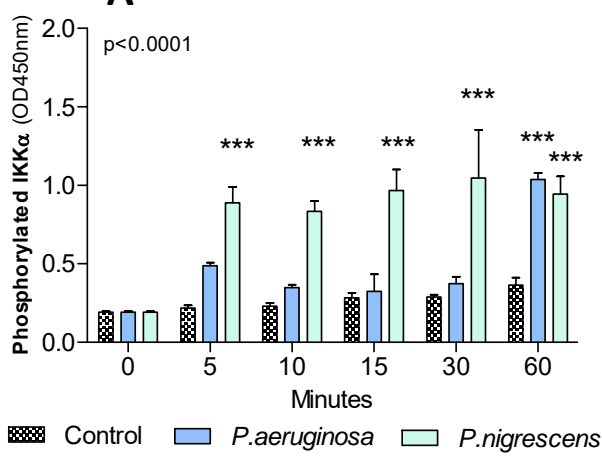

\section{B}

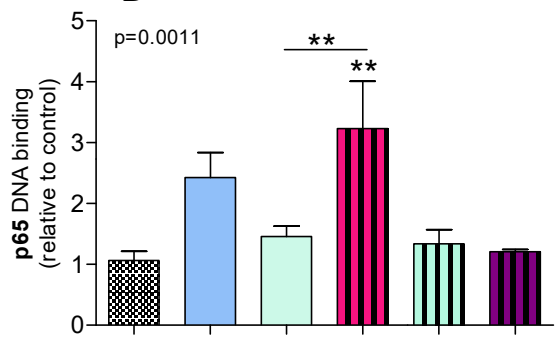

\& Control $\square$ P.aeruginosa $\square$ P.nigrescens

P.nigrescens and IKK $\alpha$ siRNA पा Scrambled siRNA

Figure 4: CFBE41o- cells infected P.aeruginosa or P.nigrescens ( $\mathrm{MOI}$ of 100) incubated under anaerobic conditions. 4A) Time course IKK $\alpha$ phosphorylation in CFBE41o- for up to 1 hour ( $n=3, * * * p<0.001,2$ way ANOVA with Bonferroni post-test). 4B) p65 DNA binding in CFBE410- cells transiently transfected with siRNA targeted against IKK $\alpha$ and infected with P.nigrescens (MOI 100) for 2 hours. Infected cell populations were compared to non-infected cell populations; siRNA knockdown populations were compared to P.nigrescens infected cells and scrambled and sham transfections were compared to nonstimulated controls ( $n=3-6, * * p<0.01,1$-way ANOVA with Bonferroni post-test).

Infection by P.nigrescens activates MAPK/p38 pathway in CF bronchial epithelial cells.

Finally, as our data strongly suggest that P.nigrescens B021S signals through TLR2 without the

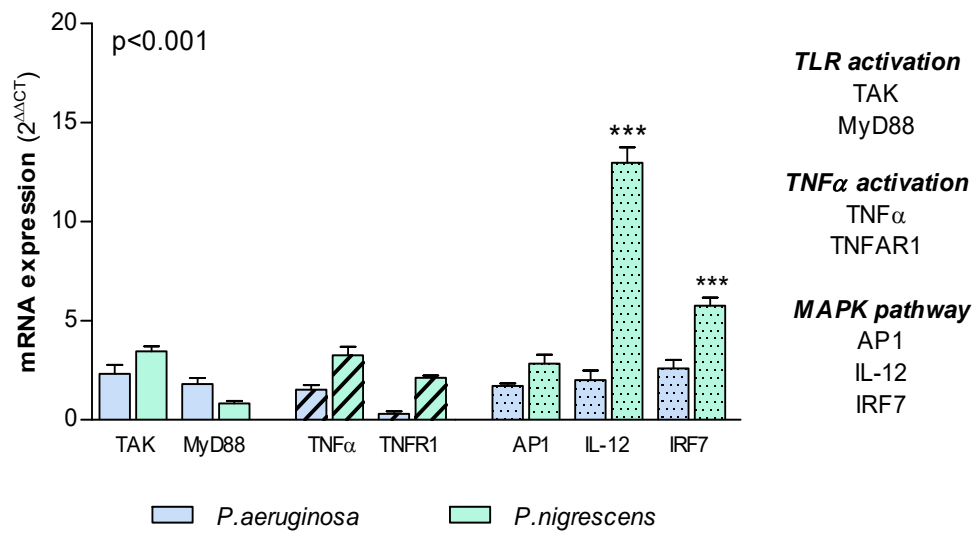

Figure 5: Gene expression (mRNA) of selected genes of the TLR and MAPK/p38 pathway 4h after infection with P.aeruginosa B021 and P.nigerscens B021S ( $n=3-5, * * * p<0.001$, 2-way ANOVA with 
248 Prevotella spp. are prevalent commensal colonizers at mucosal sites; both in the respiratory system ${ }^{27}$

249 as well as airway secretions and saliva ${ }^{28}$. Prevotella spp. have also been identified in high numbers in CF

250 airway secretions and sputum ${ }^{20,29}$, although their contribution to lung disease in PWCF through

251 changing quantity and diversity is still debated ${ }^{29,30}$. The presented study is the first to examine the

252 contribution of a clinical P.nigrescens isolate to inflammatory responses in CF epithelial cells. Our

253 clinical isolate P.nigrescens (B021S) activates TLR2 signalling resulting in a moderate induction of NF-KB

254 mediated inflammation compared to the inflammatory response from one P.aeruginosa strain (B021)

255 isolated from the same patient (Figure 3A).

256 In PWCF, infection with P.aeruginosa results in NF-KB-driven IL-8 release from bronchial epithelial cells

$257{ }^{31}$. Infection with P.nigrescens B021S also induced an increase in IL-8 gene expression in CFBE41o- cells, 258 however, at a much slower rate than in P.aeruginosa B021.

259 P.aeruginosa B021 induces NF-kB-driven inflammatory responses through a robust induction of TLR4

260 signalling in CFBE410- cells, activating transcription of inflammatory cytokines ${ }^{11}$. Infection with

261 P.nigrescens B201S significantly induced early TLR2 signalling in CFBE410- (starting 2 hours post

262 infection), and TLR5 signalling (4 hours post infection). However, our P.nigrescens strain did not utilise

263 TLR4 signalling. Furthermore, we observed a delayed and attenuated inflammatory response from

264 CFBE41o- cells (Figure 1C, 1D) compared to P.aeruginosa B021 infection, which could possibly be

265 attributed to the increased activation of IKK $\alpha$ in response to infection (Figure 4A).

266 Surprisingly, CFBE410- cells infected with P.nigrescens B021S demonstrated significantly elevated

267 levels of IL-6 gene expression at 2 hours, in contrast to the levels observed for the P.aeruginosa

268 infection (Figure 1C), indicating a different inflammatory pathway was activated in response to

269 infection with this isolate.

270 Some species of Prevotella may engage TLR2 signalling rather than TLR4 signalling and it has been

271 hypothesised that this may result in a less damaging inflammatory response and a switch to adaptive

$272 \mathrm{~T}_{\mathrm{h}} 2$ responses in vivo ${ }^{32}$. In order to confirm if infection with our P.nigrescens strain resulted in the

273 activation of TLR2 signalling rather than TLR4 signalling, HEK-293-TLR2 and HEK-293-TLR4 cells were

274 transfected with an NF-KB reporter plasmid and luciferase release from both cell types in response to

275 infection was measured over time. In this model, infection with P.nigrescens B021S induced TLR2

276 signalling rather than TLR4 signalling (Figure 2A, 2B), confirming our earlier results. In contrast to

277 P.nigrescens, infection with P.aeruginosa B021 induced the expected predominantly TLR4 signalling 
response, as measured by siRNA knockdown in CFBE410- cells, in part explaining the differences in

279 responses from the cells in response to infection with the two different bacteria.

280 We also included TLR5 (HEK-293-TLR5) into our investigations. TLR5 signalling of in vitro grown

281 flagellated P.aeruginosa (LPS smooth) is well-established ${ }^{33,34}$, but the actual pathogens in CF lung

282 disease causing chronic infections are mucoid isolates that to some degree, may have shut off flagella

283 production to avoid immune detection by the host ${ }^{34,35}$. However, up to $75 \%$ of mucoid clinical isolates

284 retain their TLR5 activating capacity during chronic CF lung infection ${ }^{36}$. The clinical isolate of

285 P.aeruginosa we studied here may still express flagella, as HEK-293-TLR5 show significant TLR5

286 signalling after infection (Figure 2C). Prevotella spp. may lack flagellin as none or only one flagella

287 synthesis pathway gene has been identified and therefore may use other structures for attachment

288 and motility ${ }^{37}$. Although we show TLR5 activation 4h after infection with P.nigrescens B021S, we did

289 not further investigate this TLR5 activation in our experimental cell model as our TLR-siRNA

290 experiments showed that NF-kB activation (p65-DNA binding) in B021S infected CFBE41o- cells was

291 due to TLR2 and not TLR5 signalling (Figure 3B, 3D). To date, flagellin is the only ligand identified for

292 TLR5 ${ }^{38}$, but TLR5 activation may also occur through non-flagellin agonists ${ }^{39}$. Therefore, the increase in

293 TLR5 activation seen here could be due to non- specific (non-flagellin) activation, for instance, through

294 residual broth in the bacterial preparations.

295 Despite a significant decrease in NF-kB activation (Figure 3B), IL-6 gene expression was not significantly

296 reduced in P.nigrescens B021S infected CFBE41o- cells with TLR2 knock down (Figure 3A), suggesting

297 that activation of other signalling pathways may contribute IL- 6 expression in response to this isolate.

298 IL-6 induction is generally regarded as NF-kB and also MAPK/p38 driven, but using LPS stimulated

299 epithelial cells of the bladder, Song et al. showed that in addition to the classical NF-KB-associated

300 pathway, TLR4 triggers a distinct and more rapid signalling response involving secondary messengers

$301 \quad \mathrm{Ca}^{2+}$, adenylyl cyclase 3-generated cAMP, and a transcriptional factor, cAMP response element-

302 binding protein). Although they did not investigate if this pathway is also activated after sole TLR2

303 engagement ${ }^{40}$, such non-NF-kB induction of IL- 6 could provide a possible explanation of our finding.

304 In order to ascertain why the inflammatory response was delayed and less robust in CFBE41o- cells

305 infected with P.nigrescens B021S when compared to the responses observed for P.aeruginosa B021

306 infection we studied phosphorylation of IKK $\alpha$ in response to infection. Studies in neuronal cells have

307 shown that IKK $\alpha$ can reduce production of inflammatory cytokines through repression of IKK $\beta$ while

$308 i k \alpha^{\mathrm{AA} / \mathrm{AA}}$ macrophages (bone marrow derived) are resistant to apoptosis and display elevated levels of

309 p65 signalling, demonstrating that IKKa can also have a regulatory effect on TLR expressing cells ${ }^{9}$. 
310 Phosphorylation of IKK $\alpha$ by NF-kB-inducing kinase (NIK) contributes to resolution of the inflammatory

311 response in macrophages ${ }^{9}$. We therefore measured phosphorylated IKK $\alpha$ in response to infection with

312 and without siRNA knockdown of IKK $\alpha$. CF bronchial epithelial cells transfected with siRNA against IKK $\alpha$

313 display significantly elevated levels of p65 DNA binding at 2 hours post infection (fold change) as

314 compared to WT controls, suggesting that sustained phosphorylation of IKK $\alpha$ in CF bronchial epithelial

315 cells contributes to resolving inflammation in response to infection.

316 Finally, as our data strongly suggest that P.nigrescens B021S signals through TLR2 activating NF-kB/p65

317 without the involvement of IKK $\alpha$ (canonical and non-canonical NF-KB pathway), we investigated the

318 MAPK/p38 pathway. MAPK/p38 activation drives IL-12-induced IFN- $\gamma$ expression in activated T cells ${ }^{41}$

319 and infected macrophages ${ }^{42}$. Our mRNA analyses shows a significant induction of IL-12 after $4 \mathrm{~h}$ of

320 stimulation with P.nigerscens B021S compared to infection with P.aeruginosa B021. Furthermore, IRF-

3217 (IFN Regulatory Factor-7) expression is also significantly increased $4 \mathrm{~h}$ after infection with P.nigresens

322 B021S. In humans dendritic cells MAPK/p38 enhances IRF-7 nuclear translocation ${ }^{43}$, therefore, IRF-7

323 expression may also be interpreted as a MAPK/p38 target. Further to this we also observed elevated

324 levels of TNF $\alpha$ mRNA as well as up-regulation of the TNF receptor TNFRSF1A (TNFR1). TNF $\alpha$ activates

325 and regulates MAPK pathways, causing both activation of MAPK3-MKKS1/2-ERK1/2, and regulation of

326 a secondary, downstream cytokine response in response to an initial stimulation with LPS. This may in

327 part explain our IL-6 data, which was not NF-KB dependent. ${ }^{55}$ Our data on IL-6 mRNA (Fig 1C) and TLR2

328 activation (Fig 2A) suggest that P.nigerscens B021S causes an earlier response ( $2 \mathrm{~h}$ ) than P.aeruginosa.

329 We only investigated the response of CFBE41o- cells to P.nigerscens B021S $4 \mathrm{~h}$ after the infection,

330 therefore, we might have missed the early upregulation of upstream TAK1 and MAPK genes AP1 and

331 MAPK14. Taken together, these data tie the early TLR2 activation by P.nigrescens B021S to the MAPK

332 pathway (Figure 5 and 6 ).

333 Microbiome studies have identified the presence of Prevotella spp. in CF sputum and some studies

334 suggest that Prevotella spp. may provoke a consistent low grade inflammatory response in the lung

335 which is profoundly less damaging to the host than the exuberant responses observed during infection

336 with other Gram-negative bacteria ${ }^{12,44}$. Further studies have correlated declining lung function and

337 elevated levels of CRP with a lower microbial diversity in the lung and reduced levels of Prevotella spp.

33845,46 . Larsen et al. proposed that the activation of TLR2 by Gram-negative anaerobic bacteria and the

339 subsequent weak inflammatory response from the host cell ensures that colonisation of the

340 respiratory system by these bacteria is tolerable for the host ${ }^{32}$. Our study supports these observations

341 as P.nigrescens B021S infection resulted in a low grade TLR2 signalling response in CF bronchial

342 epithelial cells, which is possibly less destructive and more tolerable to the host than that induced by 
343 Gram-negative P.aeruginosa B021. TLR2 signalling could also contribute to the establishment of host-

344 microbial symbiosis ${ }^{47}$, but the beneficial effect on lung immune response to infection in CF needs

345 further investigation.

346 While it appears that infection with P.nigrescens B021S may induce an inflammatory response in 347 CFBE410- cells which is less 'aggressive' than that induced by P.aeruginosa B021, we are aware that

348 the inability of the cell lines to remain under anaerobic conditions for more than 4 hours has resulted

349 in a significantly shorter experimental time-frame than other studies which assess inflammatory

350 responses to infection for up to 24 hours post infection ${ }^{48}$. Further, the reduced oxygen levels in the

351 media may have hampered the cells' ability to respond to infection, however, even under the applied

352 anaerobic conditions, the cells displayed a robust inflammatory response to infection with

353 P.aeruginosa indicating that the environment did not hinder the ability of the CFBE41o- cells to

354 respond to infection.

355 The main limitation of our study is the use of only one Prevotella strain and one P.aeruginosa strain.

356 While only one P.aeruginosa isolate was used throughout this study we also screened a range of

357 clinical P.aeruginosa isolates and compared them to the inflammatory responses obtained from

358 CFBE410- cells infected with PAO1. This was to ensure that our P.aeruginosa induced inflammatory

359 responses were representative of those published in previous studies.

360 P.nigrescens was chosen as a representative strain of many Prevotella species found in PWCF. This

361 strain was from a clinical sputum sample from a person with CF. Previous studies have investigated

362 other clinical isolates of P.aeruginosa and P.nigrescens $22,49,50$, and we aimed to compare a

363 representative strain of P.aeruginosa to a representative strain of a common Prevotella species, in this

364 case P.nigrescens.

365

366

367

368

369

370 


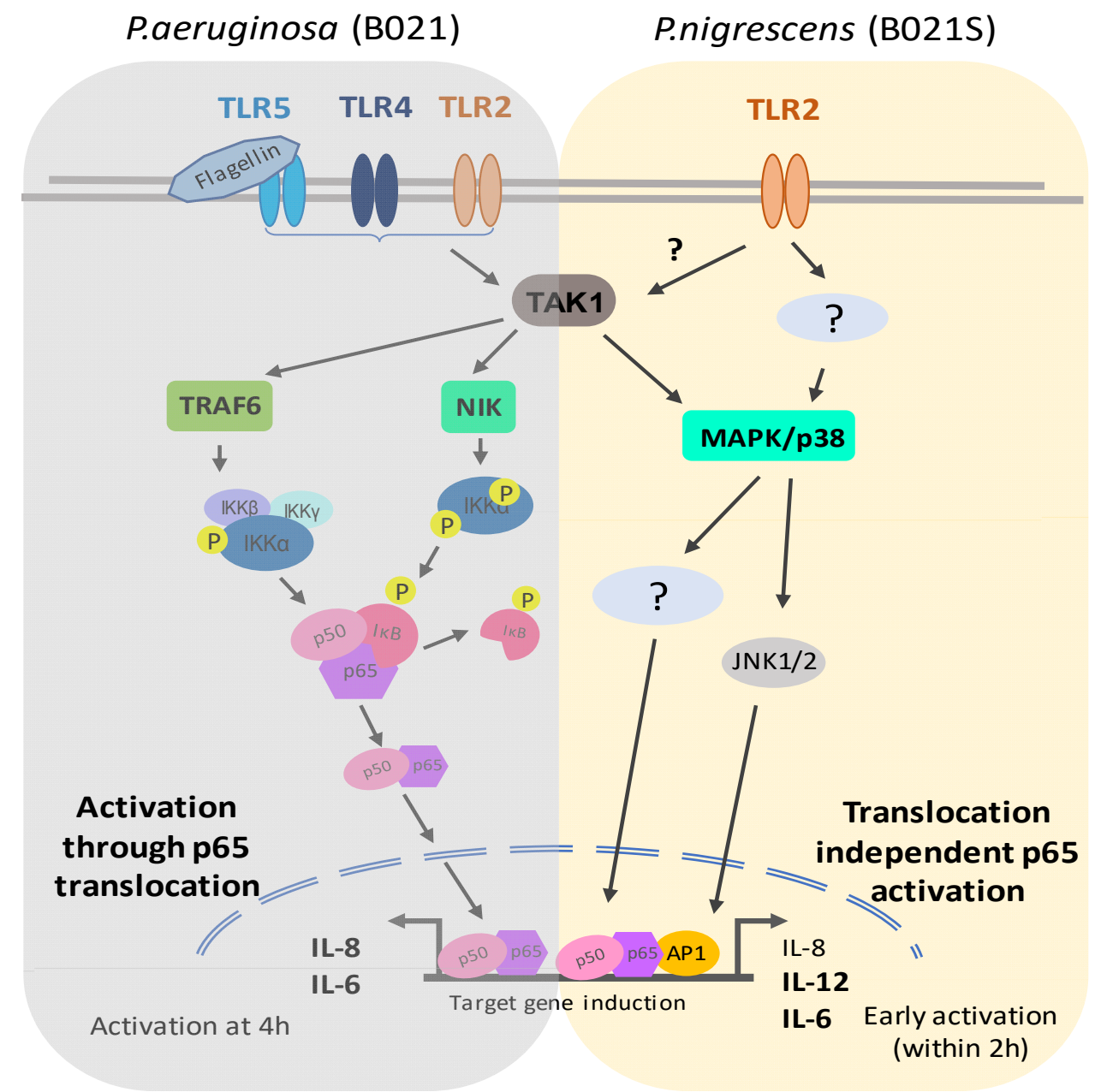

371

372

373

374

375

376

377

378

379

380

381

382

Figure 6: Schematic representation of the potential signal transduction pathways utilised by the clinical isolate P.nigrescens BO21S in comparison to the pathways used by P.aeruginosa B021 in CF airway epithelial cells.

In conclusion, there is evidence that the clinical isolate P.nigrescens B021S is associated with a lower inflammatory response in CF epithelial cells than the clinical isolate P.aeruginosa B021. Several studies have shown that Prevotella intermedia activates immune responses through the MAPK/AP1 pathway ${ }^{51,52}$ and our study suggests that P.aeruginosa B021 may also employ the MAPK/AP1 pathway. Furthermore, our data would suggest that TLR2 signalling by P.nicrescens B021S may not only activate MAPK, but also p65, even when IKK $\alpha$ is knocked down. MAPK-p38 activation lead to the activation of AP1, may also activate p65/p50 via an NF-kB translocation-independent mechanism ${ }^{53}$. While we did not explore MAPK-38 driven pathways in CFBE410- cells in response to infection with our clinical P.nigrescens isolate (B021S), our gene expression data outlined here indicate that this signalling 
387 pathway may also be activated in our cells in response to infection with P.nigrescens B021S. This

388 may in part also explain the differences observed in NF-KB driven inflammatory responses in this

389 study between the P.nigrescens B021S and P.aeruginosa B021 isolates. Figure 6 summarises the

390 potential signalling pathway of P.nigrescens B021S in comparison to the pathways used by

391 P.aeruginosa B021. However, to confirm these findings across Prevotella spp., additional

392 P.nicrescens strains need to be investigated. Furthermore, to fully understand the complex

393 inflammatory environment present in the CF lung future work will also investigate mixed infections.

396 Acknowledgements:

397 AB received a PhD studentship from the Department for Employment and Learning (DEL), Northern

398 Ireland, UK. BCS was supported by a grant from Northern Ireland Chest Heart and Stroke (NICHS,

399 2014_15).

400

401

402 Conflict of interest statement:

403 The authors have no conflict of interest, financially or otherwise.

404 
406 1. Foundation, U.C., University, J.H. \& Children, T.H.f.S. (2011).

$407 \quad 2 . \quad$ Bell, S.C., De Boeck, K. \& Amaral, M.D. New pharmacological approaches for cystic

408

409 fibrosis: promises, progress, pitfalls. Pharmacol Ther 145, 19-34 (2015).

3. Elborn, J.S. Cystic fibrosis. Lancet 388, 2519-2531 (2016).

410

411

412

413

414

415

416

417

418

419

4. Rowe, S.M. et al. Clinical mechanism of the cystic fibrosis transmembrane conductance regulator potentiator ivacaftor in G551D-mediated cystic fibrosis. Am J Respir Crit Care Med 190, 175-184 (2014).

5. Wainwright, C.E. et al. Lumacaftor-Ivacaftor in Patients with Cystic Fibrosis Homozygous for Phe508del CFTR. N Engl J Med 373, 220-231 (2015).

6. Takeda, K. \& Akira, S. TLR signaling pathways. Semin Immunol 16, 3-9 (2004).

7. Takeda, K. \& Akira, S. Toll-like receptors. Curr Protoc Immunol Chapter 14, Unit 1412 (2007).

8. Hayden, M.S., West, A.P. \& Ghosh, S. NF-kappaB and the immune response. Oncogene 25, 6758-6780 (2006).

9. Lawrence, T., Bebien, M., Liu, G.Y., Nizet, V. \& Karin, M. IKKalpha limits macrophage NF-kappaB activation and contributes to the resolution of inflammation. Nature 434, 1138-1143 (2005).

10. Oeckinghaus, A. \& Ghosh, S. The NF-kappaB family of transcription factors and its regulation. Cold Spring Harb Perspect Biol 1, a000034 (2009).

11. Buchanan, P.J., Ernst, R.K., Elborn, J.S. \& Schock, B. Role of CFTR, Pseudomonas aeruginosa and Toll-like receptors in cystic fibrosis lung inflammation. Biochem Soc Trans 37, 863-867 (2009).

12. Zhang, Z., Louboutin, J.P., Weiner, D.J., Goldberg, J.B. \& Wilson, J.M. Human airway epithelial cells sense Pseudomonas aeruginosa infection via recognition of flagellin by Toll-like receptor 5. Infect Immun 73, 7151-7160 (2005).

13. Roux, D. et al. Fitness cost of antibiotic susceptibility during bacterial infection. Sci Transl Med 7, 297 ra114 (2015).

14. Anas, A.A. et al. Lung epithelial MyD88 drives early pulmonary clearance of Pseudomonas aeruginosa by a flagellin dependent mechanism. Am J Physiol Lung Cell Mol Physiol 311, L219-228 (2016).

15. Pier, G.B. Pseudomonas aeruginosa lipopolysaccharide: a major virulence factor, initiator of inflammation and target for effective immunity. Int J Med Microbiol 297, 277-295 (2007).

16. Ernst, R.K. et al. Pseudomonas aeruginosa lipid A diversity and its recognition by Tolllike receptor 4. J Endotoxin Res 9, 395-400 (2003).

17. Pier, G.B. The challenges and promises of new therapies for cystic fibrosis. J Exp Med 209, 1235-1239 (2012).

18. Bittar, F. et al. Molecular detection of multiple emerging pathogens in sputa from cystic fibrosis patients. PLoS One 3, e2908 (2008).

19. Rogers, G.B. et al. characterization of bacterial community diversity in cystic fibrosis lung infections by use of $16 \mathrm{~s}$ ribosomal DNA terminal restriction fragment length polymorphism profiling. J Clin Microbiol 42, 5176-5183 (2004).

20. Tunney, M.M. et al. Detection of anaerobic bacteria in high numbers in sputum from patients with cystic fibrosis. Am J Respir Crit Care Med 177, 995-1001 (2008). 
21. Ulrich, M. et al. Relative contribution of Prevotella intermedia and Pseudomonas aeruginosa to lung pathology in airways of patients with cystic fibrosis. Thorax 65 , 978-984 (2010).

22. Gilpin, D.F. et al. Evidence of persistence of Prevotella spp. in the cystic fibrosis lung. J Med Microbiol (2017).

23. de Aquino, S.G. et al. Periodontal pathogens directly promote autoimmune experimental arthritis by inducing a TLR2- and IL-1-driven Th17 response. J Immunol 192, 4103-4111 (2014).

24. Jansen, H.J., Grenier, D. \& Van der Hoeven, J.S. Characterization of immunoglobulin G-degrading proteases of Prevotella intermedia and Prevotella nigrescens. Oral Microbiol Immunol 10, 138-145 (1995).

25. Yanagisawa, M., Kuriyama, T., Williams, D.W., Nakagawa, K. \& Karasawa, T. Proteinase activity of prevotella species associated with oral purulent infection. Curr Microbiol 52, 375-378 (2006).

26. Weber, A.J., Soong, G., Bryan, R., Saba, S. \& Prince, A. Activation of NF-kappaB in airway epithelial cells is dependent on CFTR trafficking and $\mathrm{Cl}$ - channel function. Am J Physiol Lung Cell Mol Physiol 281, L71-78 (2001).

27. Charlson, E.S. et al. Topographical continuity of bacterial populations in the healthy human respiratory tract. Am J Respir Crit Care Med 184, 957-963 (2011).

28. Consortium, H.M.P. Structure, function and diversity of the healthy human microbiome. Nature 486, 207-214 (2012).

29. Field, T.R., Sibley, C.D., Parkins, M.D., Rabin, H.R. \& Surette, M.G. The genus Prevotella in cystic fibrosis airways. Anaerobe 16, 337-344 (2010).

30. Fodor, A.A. et al. The adult cystic fibrosis airway microbiota is stable over time and infection type, and highly resilient to antibiotic treatment of exacerbations. PLoS One 7, e45001 (2012).

31. Kelly, C. et al. Expression of the nuclear factor-kappaB inhibitor A20 is altered in the cystic fibrosis epithelium. Eur Respir J 41, 1315-1323 (2013).

32. Larsen, J.M. et al. Chronic obstructive pulmonary disease and asthma-associated Proteobacteria, but not commensal Prevotella spp., promote Toll-like receptor 2independent lung inflammation and pathology. Immunology 144, 333-342 (2015).

33. Campodonico, V.L. et al. Evaluation of flagella and flagellin of Pseudomonas aeruginosa as vaccines. Infect Immun 78, 746-755 (2010).

34. Wolfgang, M.C., Jyot, J., Goodman, A.L., Ramphal, R. \& Lory, S. Pseudomonas aeruginosa regulates flagellin expression as part of a global response to airway fluid from cystic fibrosis patients. Proc Natl Acad Sci U S A 101, 6664-6668 (2004).

35. Faure, E., Kwong, K. \& Nguyen, D. Pseudomonas aeruginosa in Chronic Lung Infections: How to Adapt Within the Host? Front Immunol 9, 2416 (2018).

36. Blohmke, C.J. et al. Innate immunity mediated by TLR5 as a novel antiinflammatory target for cystic fibrosis lung disease. J Immunol 180, 7764-7773 (2008).

37. Ruan, Y. et al. Comparative genome analysis of Prevotella intermedia strain isolated from infected root canal reveals features related to pathogenicity and adaptation. BMC Genomics 16, 122 (2015).

38. Steiner, T.S. How flagellin and toll-like receptor 5 contribute to enteric infection. Infect Immun 75, 545-552 (2007). 
39. Das, N. et al. HMGB1 Activates Proinflammatory Signaling via TLR5 Leading to Allodynia. Cell Rep 17, 1128-1140 (2016).

40. Song, J. et al. A novel TLR4-mediated signaling pathway leading to IL-6 responses in human bladder epithelial cells. PLoS Pathog 3, e60 (2007).

41. Zhang, S. \& Kaplan, M.H. The p38 mitogen-activated protein kinase is required for IL12-induced IFN-gamma expression. J Immunol 165, 1374-1380 (2000).

42. Kim, L. et al. p38 MAPK autophosphorylation drives macrophage IL-12 production during intracellular infection. J Immunol 174, 4178-4184 (2005).

43. Wang, Q. et al. 38 MAPK is critical for nuclear translocation of IRF-7 during CpGinduced type I IFN expression in human plasmacytoid dendritic cells. J Immunol 200 (1 Supplement) (2018).

44. Bassis, C.M. et al. Analysis of the upper respiratory tract microbiotas as the source of the lung and gastric microbiotas in healthy individuals. MBio 6, e00037 (2015).

45. O'Neill, K. et al. Reduced bacterial colony count of anaerobic bacteria is associated with a worsening in lung clearance index and inflammation in cystic fibrosis. PLoS One 10, e0126980 (2015).

46. Zemanick, E.T. et al. Inflammation and airway microbiota during cystic fibrosis pulmonary exacerbations. PLoS One 8, e62917 (2013).

47. Round, J.L. et al. The Toll-like receptor 2 pathway establishes colonization by a commensal of the human microbiota. Science 332, 974-977 (2011).

48. Shahriary, C.M., Chin, T.W. \& Nussbaum, E. Respiratory epithelial cell lines exposed to anoxia produced inflammatory mediator. Anat Cell Biol 45, 221-228 (2012).

49. Einarsson, G.G. et al. Community analysis and co-occurrence patterns in airway microbial communities during health and disease. ERJ Open Research 8 (2019).

50. Sherrard, L.J. et al. Assessment of stability and fluctuations of cultured lower airway bacterial communities in people with cystic fibrosis. J Cyst Fibros, S15691993(1519)30052-30059 (2019).

51. Guan, S.M. et al. Prevotella intermedia induces matrix metalloproteinase-9 expression in human periodontal ligament cells. FEMS Microbiol Lett 283, 47-53 (2008).

52. Kim, S.J. et al. Prevotella intermedia lipopolysaccharide stimulates release of tumor necrosis factor-alpha through mitogen-activated protein kinase signaling pathways in monocyte-derived macrophages. FEMS Immunol Med Microbiol 51, 407-413 (2007).

53. Shuto, T. et al. Activation of NF-kappa B by nontypeable Hemophilus influenzae is mediated by toll-like receptor 2-TAK1-dependent NIK-IKK alpha /beta-I kappa B alpha and MKK3/6-p38 MAP kinase signaling pathways in epithelial cells. Proc Natl Acad Sci U S A 98, 8774-8779 (2001). 